\title{
NIST Certification of Metallurgical Standard Reference Materials for Microanalysis - A Short History
}

\author{
R. B. Marinenko
}

National Institute of Standards and Technology (NIST), MS 8371, Gaithersburg, MD 20899

The development of the electron microprobe in the 1950's followed by the availability of commercial instruments in the early 1960's had a serious impact on metallurgy. With the new instrument, scientists could extend microscopic interpretations to determining the chemical composition of each phase because the excitation volume of the electron beam was only about 1-3 $\mu \mathrm{m}$, less than single phase dimensions that were usually several microns. In addition, changes in element concentrations across phases and boundaries could be characterized.

To define concentrations and trends in quantitative terms, electron probe microanalysis (EPMA) requires reference standards to compare the characteristic x-rays emitted from the unknown to that of a material with a know concentration. For metals, pure elements can be used and are often readily available with some exceptions, such as gallium. For some metals, the purification process may fail to remove minor or trace amounts of other elements like vanadium in titanium. Other pure elements may oxidize readily as in the case of the rare earths elements. In such cases stoichiometric compounds, if available, may be used to replace the need of a pure element. Elemental analysis can sometimes be influenced significantly by the matrix in which the element of interest occurs, necessitating a standard similar in composition to the unknown, thus limiting the uncertainties introduced by the atomic number, x-ray absorption, and fluorescence effects of the matrix in the corrections procedures.

For these reasons and because of the need to test EPMA matrix correction procedures, that were also being developed in the 1960's, microanalysis reference standards with two or more elements were needed. Such reference standards had to have little or no heterogeneity on the $\mu \mathrm{m}$ scale so that the elemental composition of any point would be representative of the bulk material. The early work on such microanalysis reference standards at the National Bureau of Standards (NBS, the former name of NIST), began on metal alloys that had already been certified for bulk composition as NBS SRMs (Standard Reference Materials) for spectrochemical analyses [1]. With EPMA, the composition of inclusions were determined and the extent of element heterogeneity in the matrix of low-alloy steels and wrought and chill-cast brass alloys were studied to determine if these materials could be useful reference standards for EPMA.

Later, a tungsten-20\% molybdenum alloy was prepared via a powder metallurgy process specifically for use as a microanalysis reference standard [2]. Since it was difficult to prepare single-phase metal alloys, there was significant interest in the use of powder metallurgy for the preparation of microanalysis standards with minimum heterogeneity. The W-20Mo alloy was successful although some heterogeneity was observed in the alloy, yet further attempts with powder metallurgy were not made at NIST. This SRM is still available.

Two alloys of iron 3\% silicon, a commercially available sheet stock (SRM 483) and NBS SRM 1134 were tested for micro-heterogeneity is 1970 , for the first time using automated sampling and data 
acquisition techniques with the energy dispersive (EDS) detector instead of wavelength dispersive spectrometry that is conventionally used in heterogeneity testing [3]. Several representative specimens were studied and sampled in arrays of 400 points that were repeated in some tests. The coefficient of variation was conservatively estimated to be $1 \%$ or less for the entire set of specimens. Unfortunately, SRM 483 is no longer in stock, but SRM 1134 is available.

The gold-silver and gold-copper alloys (NBS SRMs 481 and 482) are probably the best known and most used microanalysis SRMs developed at NBS [4]. Both sets include the pure end members with intermediate nominal compositions of $0.2,0.4,0.6$, and 0.8 mass fraction of the second element. They were specifically designed for evaluating matrix correction procedures although they have proved to be valuable for routine quality control procedures and EDS calibrations over time. The materials were prepared commercially for NBS, and they were evaluated by NBS for heterogeneity and elemental composition. These SRMs are still available. Recent studies of SRM 482 show that small copper oxide inclusions are present in some of the alloys, but these should not effect the certified composition values.

The most recent alloy issued for microanalysis was the austenitic iron-chromium-nickel alloy (nominal mass fraction composition of $0.71 \mathrm{Fe}, 0.11 \mathrm{Ni}$, and $0.18 \mathrm{Cr}$ ) issued in 1972 and reissued in $1980[5,6]$. The uncertainties due to heterogeneity at the expanded $2 \sigma$ level was $2 \%$ or less. For the 1980 issue, rather than simply using standard deviations and coefficients of variation, a more comprehensive statistical sampling and analysis procedure was developed to enable calculation of the between points, between specimens, and experimental uncertainties that contributed to heterogeneity. With a few modification, we continue to use this procedure today and it has been written into an ISO document that is close to becoming a final standard.

In the late 1970s, through the 1980s and 1990s, glasses were issued as microanalysis SRMs because a large number of elements in trace and minor concentrations could be incorporated into glasses without disturbing the homogeneity of the glass, and NIST had a group that was very good at producing glasses with different matices. The heterogeneity of all SRMs were reevaluated with periodic integrator traces and reported in 1979 [7].

Soon we expect to issue another alloy, $\operatorname{TiAl}(\mathrm{W}, \mathrm{Nb})$, that is used in the aircraft industry. It will be the first metal alloy to be issued as a microanalysis SRM for many years. Ti and Al, the major constituents, show little heterogeneity while $\mathrm{W}$ and $\mathrm{Nb}$ that are minor constituents, are heterogeneous by several percent relative.

\section{References}

1. H. Yakowitz et. al., NBS Spec. Pub. 260-10 (1965).

2. H. Yakowitz et. al., NBS Spec. Pub. 260-16 (1969).

3. H. Yakowitz et. al., NBS Spec. Pub. 260-22 (1971).

4. K.F.J. Heinrich et. al., NBS Spec. Pub. 260-28 (1971).

5. H. Yakowitz et. al., NBS Spec. Pub. 260-43 (1972).

6. R.B. Marinenko et. al., NBS Spec. Pub. 260-70 (1981).

7. R.B. Marinenko et. al., NBS Spec. Pub. 260-65(1979).

All references on web site http://patapsco.nist.gov/srmcatalog/sp_publications/publications.htm 